through the circuit. Thus if the system of longitudinal magnetic waves inside the core were established, it would produce the system of transverse waves around it.

\section{FLow OF ENERGy}

The rate of flow of energy through unit area at right angles to $\rho$ is by Poynting's theorem proportional to the product $Q \gamma$. As $\gamma$ vanishes outside the core, there is no radial flow of energy and hence no leakage of energy as the photon passes through space. The energy in the wave front will be concentrated round the axes. On the axis itself both $Q$ and $\alpha$ vanish and only the longitudinal magnetic force is left. As we recede from the axis the amplitude of $Q$ and $\alpha$ increase until the boundary of the core is reached; here both have their maximum values and continually diminish as the distance from the axis increases, the energy per unit area of the wave front varying inversely as the square of the distance from the axis. Thus the radiation may practically be regarded as confined within a cylindrical pencil and not as extending throughout space.

The train of waves emitted from a luminous atom will be of finite length, and the amplitude of vibration will not be constant along the train, while those to which we have applied the mathematics are infinitely long trains of constant amplitude. We know, however, by Fourier's theorem that we can regard the first type of waves as the sum of a number of those of the second, and the results we have obtained will apply to each constituent of this sum.

Owing to the symmetry in Maxwell's equations between the electric and magnetic forces, a system of rings of magnetic force would possess the properties of the system of lines of electric force which we have been considering.

Trinity Lodge,

Cambridge. Jan. 27.

\section{J. J. Thomson.}

The Ceremonial Dedication of the Philipp-LenardInstitut at Heidelberg

The description given in NATURE of January 18 of the proceedings at the ceremonial dedication of the Philipp-Lenard-Institut at Heidelberg must form sad reading for the general body of scientific workers throughout the world, and more especially for those of us who owe so much to the stimulating instruction and courteous hospitality which we had the privilege to enjoy as students at one or other of the German universities in the past. In reading the almost incredible utterances of some of the present representatives of academic Germany on the occasion of this ceremonial at Heideiberg, it has occurred to me that in our amazement at such extravagances we are inclined to overlook some of the stark realities of the situation, and that perhaps they were these which either consciously or instinctively led the speakers to give expression to the wild and unbalanced statements which they are reported to have made.

In the mass-hysteria accompanying all revolutionary movements it is invariably the case that some group-associations in the country are singled out for sttack and submitted to drastic and exemplary punishment or even total suppression by the new and self-created authorities, as we see in the case of the persecution of the Jewish population in Germany to-day. It was, therefore, by no means improbable that the particular group of the intelligentsia represented by the universities might at any moment be destined to meet with a similar fate, in which the whole of their organisation and activities might be proscribed and swept away at the behest of the new masters in the State. To avert the possibility of such a dire calamity, it is not surprising that there should be found members of the group in question ready to come forward and give expression to assurances of complete sympathy with even the most extreme and unreasonable views and actions of the new rulers.

To us in Britain, who fortunately are not at the moment in danger of becoming the victims of masshysteria, such assurances appear as a deplorable negation of some of the most fundamental and sacrosanct articles of our scientific credo. Our less fortunate German colleagues, on the other hand, would seem to be faced with much the same situation as was Galileo cited before the Inquisition, and that some of them have had recourse to the opportunist tactics of Henry of Navarre when he declared that "Paris is worth a Mass".

Jan. 29.

P. F. F.

\section{Deuterium as an Indicator in Fat Metatolism}

Ix has hitherto not been a simple matter to deter. mine whether the fatty acids in lipines such as lecithin, which are constituents of all active animal cells, play a part in the normal metabolism of fats. One reason for this is that suitable methods for 'labelling' fats which could be administered in the diet and then traced in the body have not been available. With the advent of deuterium a method for marking fats and using them in this way has become possible.

With the object of determining whether the fatty acids which are components of the fats of the diet can be found after absorption combined as lipines in various organs, we have partially saturated linseed oil with deuterium, and administered $2 \mathrm{gm}$. of the oil daily to an adult rat for seven days. The oil contained deuterium amounting to 4.9 per cent by weight of its total hydrogen content. The rat was then killed, and the ether-soluble substances of the liver and kidneys separated by the acetone method into lipine and glyceride fractions. A sample of fat from adipose tissue in the abdomen was also obtained. Each fraction was then examined for deuterium by burning it in a suitable combustion furnace, collecting the water produced and determining its density. The following figures for the deuterium content as percentage of total hydrogen were obtained :

Liver lipines, 0.98 ; liver glycerides, 0.97 ; kidney lipines, $0 \cdot 63$; adipose tissue fat, $0 \cdot 75$.

An estimation of the fat in the frecs, passed during the experiment, showed that absorption of the deuterium-containing fat was almost complete. These results show that the fatty acids containing deuterium have entered largely into the lipines both in the liver and kidney. The natural inference is that the lipines, although they may be important as structural elements in the cell, are probably also actively concerned in the metabolism of fats.

Whilst this work was in progress, Sinclair' ${ }^{1}$, using elaidic acid for 'marking' purposes, has also shown that the lipines of the liver take up fatty acids which have recently been administered in the food. 